intervals between the spasms he was unable to separate the teeth more than half an inch, but he could swallow raids without great difficulty. He was ordered bromide of potassium and chloral hydrate mixture--fifteen grains of aach every four hours. This was continued during two days without benefit, the spasms becoming more frequent and more severe. Owing to the great difficulty in swallowing it was then stopped. On Oct. 27th injections of antitoxin were commenced. This was very kindly forwarded to Leicester by Dr. Roux of the Institut Pasteur, Paris. The total amount used was fifteen grammes of dried serum, dissolved in ten times its weight of distilled sterilised water ; 100 fluid grammes were injected in the first twentyfour hours, fifty at 6 P.M. and fifty at 11 A.M. On each occasion chloroform was administered, the fluid being injected subcutaneously with a sterilised syringe in very many punctures in the thighs, buttocks, arms and chest. In the second twenty-four hours the remaining fifty grammes were injected in two parts at 830 P.M. and 5.30 P.M. The punctures determined a slight and transient redness of the skin at the points of puncture. No pain was complained of on recovering from the effects of the anæsthetic. On the second day of injection the convulsions were noticeably diminished in severity, the lividity and the clenching of the teeth being less marked, as well as the arching of the back. From this time there was a slow and gradual abatement of the severity of the spasms, though they did not entirely cease for another week; their irequency was also very much less. On Oct. 30 th he again took fifteen grains of bromide of potassium every four bours and continued to do so during one week. The improvement was undoubtedly simultaneous with the injections, and the severity of the spasms was less from the day they were commenced. It is only reasonable to ascribe the benefit to the antitoxin, as the drugs appeared to have little or no effect. As regards the local treatment, on the day after admission the thumb-nail was removed under chloroform and the suppurating matrix freely incised and dressed with fomentations of a 1 in 20 carbolic acid solution. It is remarkable how little constitutional disturbance was caused by the injections of such large quantities of serum, and the absence of constitutional disturbance shows the thorough asepsis of the preparation and preservation of the serum. The only elevation of cemperature was on the second day, when it reached $100^{\circ} \mathrm{F}$.

\section{SOUTH DEVON AND EAST CORNWALL HOSPITAL.}

A CASE OF CEREBRAL H YEARS OF AGE, FOLLOWING MITRAL DISEASE.

\section{(Under the care of Dr. Fox.)}

CEREBRAL hæmorrhage in children is an unusual occurrence, and in this case the symptoms were possibly dependent partly on the thrombosis of the sinus and veins, for the clot was of small size, the influence of age and the changes in the vascular system which it produces are well known, and we "very rarely find cerebra! hamorrhage in a patient under twenty years of age.

On Oct. 24th, 1893, a child seven years of age was admitted to the South Devon and East Cornwall Hospital suffering from mitral disease. There was some ascites and also dropsy of the legs. Early in November the quantity of urine passed by the patient became very small, and the child was at Grst frettul, then drowsy, and finally quite comatose. There were lucid intervals. In a day or two the child was seized with convulsive attacks, which were left-sided. The head was turned towards the left, and there was conjugate deviation of the eyeballs in the same direction. There were violent twitchings of the left side of the face, particularly of the lower muscles and of the left arm. The legs were not much affected. The left leg twitched very slightly. The pupils were dilated and the pulse was rather hard. A hot bath always put an end to these attacks. The patient died and a post-mortem examination was made. The vessels running in the pia mater about the upper part of the right fissure of Rolando, the tongitudinal sinus, and some veins over the cerebellum were thrombosed. About half an inch below the surface of the brain, at the upper part of the right fissure of Rolando, there was a round dark clot the size of a large cherry.

Remarks - It is worthy of note that although the clotting in the vessels, which by pressure on the cortex must have caused the convulsions, took place most thoroughly at the upper part of the motor area, the leg was very little affected. This could be easily accounted for by the pressure on, and tearing of, the nerve fibres on their way to the leg, which was brought about by the rupture of a ressel and the formation of the clot.

\section{Attedical Soxtieties.}

\section{ROYAL MEDICAL AND CHIRURGICAL SOCIETY.}

\section{Spontaneous Disappearance of Solid Abdominal Tumours.}

AN ordinary meeting of this society was held on Jan. 23rd, Dr. CHURCH, Senior Vice-President, being in the chair.

Mr. J. GReig Smith (Bristol) read a paper on the So-called Spontaneous Disappearance of Solid Abdominal Tumours. Three cases were recorded, the common leading features in which were the presence of a solid tumour in the abdomen, absence of pyrexia, clinical evidence of malignancy on abdominal section, and ultimate disappearance of the tumour, with complete restoration to health. The first case was that of a young man aged twenty-five. Intestinal obstruction existed due to a solid tumour as large as a cocoanut situated in the lower abdomen. The tumour, being on inspection diagnosed as malignant and being adherent to intestines, was not disturbed. Enterostomy was performed for the obstruction. The tumour slowly diminished in size, and six months later, when the abdomen was opened for the performance of enterorrhaphy, it was found to have disappeared. The patient, after four years and a half, continued in good health. In the second case, that of a woman aged fifty-five, the tumour lay in the umbilical region, and was adherent at and around the umbilicus. It was globular in shape and as iarge as a child's head at birth. On abdominal section the intestines were found to be adherent to its surface on one side. Diffusing malignancy was diagnosed, and no attempt at removal was made. A small collection of muco-pus at the umbilicus was evacuated, and the slight discharge from a fistula which formed there continued for over two years. The tumour disappeared, the sinus closed, and the patient was now quite well. A girl aged nineteen was the subject of the third case. Here the tumour was as large as an eight months' pregnant uterus, filling and distending the upper and right side of the abdomen, but not dipping into the pelvis. On abdominal section the tumour was diagnosed as malignant and unremovable. A fæcal fistula formed; the tumour gradually diminished in size and ultimately disappeared. The intestinal opening was closed by operation, and the patient vias at present in good health. Comparative observations were made on solid tumours of myomatous, tuberculous, and inflammatory nature, which in the writer's experience had been found to disappear after operation. An explanation as to the origin of the tumours in question was made to rest on the process of phagocytosis and the heaping up of embryonic protective cells around a minute fistulons opening communicating with intestine. Cure was accidental, but not spontaneous, depending in one case on a diversion of intestinal contents and in the two others on an external opening of the fistula.

Dr. CHCRCH remarked that it was just in such cases as those under discussion that the quacks made capital. He suggested that some inflammatory lesion or enlargement of a Meckel's diverticulum might be the cause of some instances. An accumulation in a diverticulum, by preventing the passage of fæces along the intestine, would gradually lead to an increase of its own contents.

Mr. Alban Doran said that the paper dealt with the very delicate subject of the erroneous or doubtful diagnosis of abdominal tumours. In cases of chronic peritonitis, accompanied by adhesions of intestine and by the accumulation of scybala, clinical signs might arise which closely resembled those of solid tumour. Chronic tuberculous peritonitis was not rare in tolerably elderly people, and as a result masses of inflammatory material might be formed on the surface of the intestine; these might subsequently disappear, and the temperature might remain for long periods little if any above the normal. Such inflammatory formations simulated a semi-solid or semi-cystic tumour and were 